\title{
Research on Two-stage Order Picking Sequencing for Intensive Shelf
}

\author{
Xue Tian ${ }^{1, a}$, Li Zhou ${ }^{2}$ and Jianglong Yang ${ }^{3}$ \\ ${ }^{1}$ Associate Professor of Beijing Wuzi University, China; Visiting Assistant Professor in CSUSB, California State University, San Bernardino, \\ USA \\ ${ }^{2}$ Professor of Beijing Wuzi University \\ ${ }^{3}$ Student of Capital University of Economics and Business
}

\begin{abstract}
The increasingly usage of Intensive shelves, greatly increase the utilization of storage space, but also is more demanding on order picking time. Based on the storage layout of intensive mobile shelves, this paper combines the time cost of shelf movement with the moving distance under the guidance of seeking global optimum. Transform the single order picking process into TSP problem, while considering the picking process. The waiting cost of the order, and the minimum of the picking cost and the waiting cost of the whole batch of orders requiring shelf movement, to establish a two-stage mathematical model of the order picking order. Then, the algorithm for solving the model is designed, and the simulation is carried out by numerical examples to illustrate the law and characteristics of the problem more vividly, in order to provide reference and reference for the order picking activities of intensive mobile shelves.
\end{abstract}

\section{Introduction}

In the current era, with the continuous development of e-commerce and urban distribution, the efficiency of logistics activities has also been more and more important. As a core function of logistics activities, warehouse activities have attracted much attention. As far as order picking in warehouse activities is concerned, it has long been recognized as one of the most time-consuming activities in warehouse activities, and is also an important bottleneck for improving the efficiency of warehousing activities. At the same time, due to the increasingly scarce characteristics of land resources in urban areas, the storage cost of goods is rising rapidly, which requires the storage area to increase the utilization of warehouse volume as much as possible. As a result, a more intensive storage shelf layout has emerged in which intensive mobile shelves have emerged and are increasingly being applied to the practice of warehouse activities. Although the intensive mobile shelf can improve the utilization of the storage space of the warehouse, it needs to remove the goods by continuously moving the shelves during the order picking process, which in turn further aggravates the time consuming of the order picking activities, even the shelf moving place. It takes much longer than the time to take the goods out of the shelf, resulting in a reduction in the efficiency of the storage operation to some extent.

Despite this, in some cases (for example, the rent of the warehouse location is much higher than the time cost), the use of intensive mobile shelves for warehouse layout to improve inventory space utilization is still the choice

\footnotetext{
a Corresponding author: xue.tian@csusb.edu
}

of some enterprises. Then, how to further improving order picking and improve picking efficiency has become an important issue for enterprises to think about.

\section{Introduction to the status of intensive mobile shelves}

\subsection{Intensive mobile shelves use status}

\subsubsection{Introduction to intensive mobile shelves}

Intensive mobile shelves, also known as dense shelves or dense cabinets, are densely stored cabinets or shelves. Because they are removable and densely arranged, they can save a lot of storage space, as shown in Figure 1.

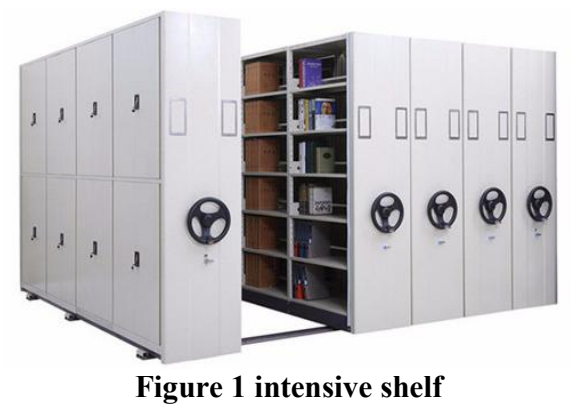

The intensive mobile shelves are arranged according to the characteristics of the warehouse, and the space used. The multi-section and multi-connection are located on a fixed track to form a whole body. The columns are 
accessed by hand or electric, and the operation is easy and flexible, and the operation is stable ${ }^{[1]}$. The contact surfaces between the dense mobile shelf frame is cushioned and magnetically sealed. There is a dustproof plate at the top and a mouse-proof device at the bottom. It is good dust, mouse, moisture and fire resistance.

\subsubsection{Intensive mobile shelf structure}

Generally speaking, the shelves are generally composed of cabinets, rails, transmission systems, etc. (see Figure 1), as follows:

(1) cabinet body includes: column, partition, hanging plate, hanging piece, side plate, top plate, dividing rod, bottom frame, door panel;

(2) transmission system includes: bottom rail bearing, cast iron angle wheel, transmission chain, transmission wheel, brake steering wheel or rocker;

(3) Tracks include: rail seat, solid core steel, and antidumping device at the track end;

(4) Other accessories: seal strip between columns, dust plate at the top, brake switch, total lock, label holder, etc.

\subsubsection{Intensive mobile shelf types}

(1) Classification by storage items: intensive archives, dense file shelves, dense data shelves, dense map bookshelves, dense tape racks, intensive medical records, dense blueprints, dense base frames, dense specimen racks, and dense sample racks;

(2) Classification by storage location: archive room intensive cabinet, data room intensive cabinet, library room intensive cabinet, storage room intensive cabinet, archive intensive cabinet, data warehouse intensive cabinet, warehouse intensive cabinet;

(3) Classified by unit of use: archives intensive cabinets, hospital intensive cabinets, densely populated cabinets, enterprise intensive cabinets, school intensive cabinets, design institute intensive cabinets, insurance company intensive cabinets, bank intensive cabinets, supermarket intensive cabinets;

(4) Classification by the production process: no door dense cabinet, semi-closed dense cabinet, fully enclosed dense cabinet, two column type dense cabinet, three column type dense cabinet, box type dense cabinet.

\subsection{Research status of intensive mobile shelves}

\subsubsection{Research on optimization strategy of warehouse picking system}

The existing warehouse picking mode can improve the flexibility of warehouse to a certain extent, but there are inevitably some problems with various types of warehousing picking modes, which has led to some scholars' relevant research.

BoysenN et al. (2017) explored the problem of order picking queues for mobile shelf warehouses, and reduced the time required to move heavy shelves by optimizing the order queueing, and enhanced picking efficiency; $\mathrm{HeZ}$ et al. (2018) in the context of intelligent warehouse automation services, provided a differentiated probability queuing strategy for serving customer orders using the AGV system, which enables to solve the joint optimization problem and reduced the processing time by 19.64\%; RoyD et al. (2019) established the mobile fulfillment system based on the multi-class closed queuing network model.

\subsubsection{The development of the flexible storage system}

The development of the warehousing system highlighted features such like compact, intelligent, economic, efficient, stable, and easy to expand ${ }^{[2]}$. The warehouse layout is developed from wide-channel $\mathrm{g}$ to narrow-channel, non-channel-to-person picking mode. the selection of picking equipment is changed from forklift, hydraulic handling, paper guiding to sound and light guidance, electronic label, intelligent conveyor belt, AGV intelligent equipment etc.

\section{Intensive mobile shelf picking problem}

\subsection{Warehousing layout assumptions}

Suppose there is a warehouse with a set of mobile dense shelves. As shown in Figure 2, the aisles between each rows of shelves can be opened, indicated as $A_{i}(i=1,2, \ldots \ldots n)$.

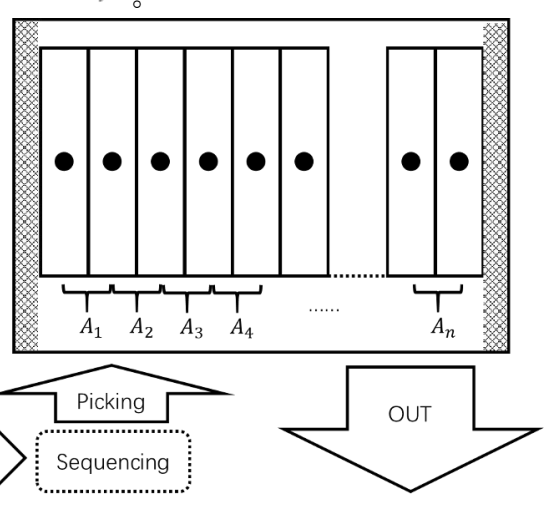

Figure 2 work process of intensive shelf

The time taken to open each aisle is represented by $D_{i j}$ $(i, j=0,2, \ldots n)$, which is the time it takes for opening the aisle from the $\mathrm{i}$ position to the $\mathrm{j}$ position. Assuming that the time cost per channel of moving a row of shelves is $v$, the time cost of all the moving times can be expressed as a table related to $\mathrm{v}$, see Table 1 .

Table 1 cost of all the moving times

\begin{tabular}{|c|c|c|c|c|c|c|}
\hline & \multirow{2}{*}{$D_{i j}$} & \multicolumn{5}{|c|}{$\mathbf{j}$} \\
\hline & & 1 & 2 & 3 & $\ldots$ & $\mathrm{n}$ \\
\hline \multirow{5}{*}{ i } & 1 & 0 & $\mathrm{~V}$ & $2 \mathrm{v}$ & $\ldots$ & $(n-1) v$ \\
\hline & 2 & $\mathrm{~V}$ & 0 & $\mathrm{~V}$ & $\ldots$ & $(n-2) v$ \\
\hline & 3 & $\mathrm{~V}$ & $\mathrm{~V}$ & 0 & $\ldots$ & $(n-3) v$ \\
\hline & $\cdots$ & $\cdots$ & $\cdots$ & $\ldots$ & $\ldots$ & $\cdots$ \\
\hline & $\mathrm{n}$ & $(n-1) v$ & $(n-2) v$ & $(n-3) v$ & $\ldots$ & 0 \\
\hline
\end{tabular}




\subsection{Order picking hypothesis}

Assume we need to pick a batch of orders within a certain time requirement. the goods picking time is affected by the moving shelf status and the order contents $^{[3]}$. the entire batch of orders is already sorted and the picker is arranged to pick the goods with a certain sequencing. there is only one picker and one mobile channel in our model.

Suppose that in a certain period, the number of orders received is $m$, and $B$ is used to represent the order, then there are $\mathrm{B}=\left\{B_{1}, B_{2}, \cdots, B_{m}\right\}$. Concerned the goods in each order and location of each shelf, the aisle needs to be opened can be determined. That is, the mobile aisle corresponding to the order $B_{d}$ needs to be opened, which can be derived from the following collection:

$$
\left(E_{d 1}, E_{d 2}, \cdots, E_{d n}\right)
$$

Where $E_{d j}=0$ or $1 ; \mathrm{d}=1,2, \ldots \mathrm{m} ; \mathrm{j}=1,2, \ldots \mathrm{n}$. When $E_{d j}=0$ the related aisle $A_{j}$ does not need to be opened; when $E_{d j}=1$, the related aisle $A_{j}$ needs to be opened. See Table 2 for the mobile aisle that need to be opened for $m$ orders in the certain period.

Table 2 mobile aisle need to be opened

\begin{tabular}{c|c|c|c|c}
\hline & $A_{1}$ & $A_{2}$ & $\ldots \ldots$ & $A_{n}$ \\
\hline$B_{1}$ & $E_{11}$ & $E_{12}$ & $\ldots \ldots$ & $E_{1 n}$ \\
\hline$B_{2}$ & $E_{21}$ & $E_{22}$ & $\ldots \ldots$ & $E_{2 n}$ \\
\hline$\ldots \ldots$ & $\ldots \ldots$ & $\ldots \ldots$ & $\ldots \ldots$ & $\ldots \ldots$ \\
\hline$B_{m}$ & $E_{m 1}$ & $E_{m 2}$ & $\ldots \ldots$ & $E_{m n}$ \\
\hline
\end{tabular}

Thus, the relationship between the aisle that need to be opened by multiple orders can be further analyzed. thereby coordinating the ordering arrangement of all the orders in the entire on period.

\section{Two-stage model of intensive mobile shelf order picking}

Since the time cost of moving shelf is much higher than the picking cost, in the model picking time is not considered. Also, the orders follow a FCFS rules, so the later orders will generate waiting costs before is actually picked $^{[4]}$. The longer the waiting time cost, the lower customer service level. Therefore, when establishing the model, it is necessary to consider both the time cost of moving the shelf and the time cost of order waiting ${ }^{[5]}$. A comprehensive balance is required to minimize the sum of the total time cost.

\subsection{Stage 1: Finding the minimum time cost for a single order}

First consider the time cost of a single order $B_{d}$. From the previous assumptions it is clarified the time cost of the aisle movement is linearly and positively related to the moving distance. Therefore, each aisle needs to be opened can be regarded as a point, and the whole process of picking for order ${ }^{B}$ can be token as a TSP problem. We use $Y_{n}$ to stand for the location of each shelf. As shown in Figure $3, A_{1}, A_{2} \cdots A_{n}$ are different location of the shelves, and the specific distances between any two shelves are shown in Table 1. The initial position of the moving channel is $A_{j^{0}}$. In addition, let $A_{0}$ be a virtual point, the distance from $A_{0}$ to the initial position $A_{j^{0}}$ is 0 . The distance from $A_{0}$ to other points is equal to the distance from the initial position $A_{i}{ }^{0}$ to other point, and it is 0 . In addition, since each order needs to open different channels, if an order needs to open all channels, its network diagram is the whole picture of Figure 3. If an order only needs to open some channels, its network diagram is just part of Figure 3. Therefore, when the channel needs to be opened according to different orders is determined (see Table 1), the network diagram can also be determined.

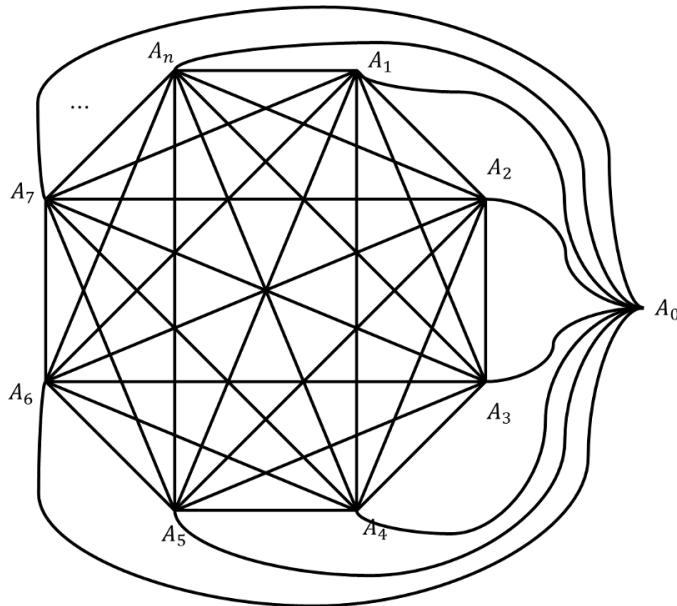

Figure 3 schematic diagram of TSP problem

Let $F_{i j}$ indicate whether to move from channel $A_{i}$ to channel $A_{j}$ according to order $B_{d} . D_{i j}$ stands for the cost per unit distance needs to be moved. Then, under the previous assumptions, we could establish a model like the following which shows that the target of the single stage optimization is lowest time cost.

$$
\begin{gathered}
F_{i j}=\left\{\begin{array}{c}
1 \quad \text { from } \mathrm{i} \text { to } \mathrm{j} \\
0 \quad \text { no need from } \mathrm{i} \text { to } \mathrm{j}
\end{array} \text { where, } i, j=1,2 \cdots n\right. \\
\min Z_{d}=\sum_{i=1}^{n} \sum_{j=1}^{n} D_{i j} F_{i j} \\
\text { s.t. }\left\{\begin{array}{c}
\sum_{i=1}^{n} F_{i j}=1 \quad j=1,2, \cdots n \\
\sum_{j=1}^{n} F_{i j}=1 \quad i=1,2, \cdots n \\
F_{i j}+F_{j i} \leq 1 \quad i \neq j \\
F_{i j}+F_{j k}+F_{k i} \leq 2 \quad i \neq k \neq j \\
\vdots \\
F_{i j}+F_{j k}+F_{k l}+\cdots+F_{p i} \leq n-1 \\
i \neq k \neq \cdots \neq p \neq j \\
F_{i j}=0 \text { or } 1 \quad i, j=1,2 \cdots n
\end{array}\right.
\end{gathered}
$$

\subsection{Stage 2: find the minimum time cost of a batch of orders}

After the start of the picking process, what the waiting cost of the order, which has not been picked up, needs to increase is the time taken for the previous order picking. The $d_{s}$ indicates that the order numbered $\mathrm{d}$ is picked at the $S_{\text {th }}$ time, $Z_{d_{n}}$ indicates the picking time of the order $B_{d}$.

It can be seen that after the picking of an order is

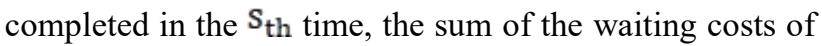


the remaining (m-s) orders is increased by $(\mathrm{m}-\mathrm{s}) Z_{d_{1}}$. To complete $\mathrm{m}$ order picking during the entire picking process, the sum of all order waiting costs is $\sum_{s=1}^{m}(m-s) Z_{d_{s}}$

The overall goal of this problem is to find that the sum of the picking cost and the waiting cost of all the picking orders in the inspection cycle is the smallest.

$$
\begin{gathered}
\min W=\sum_{s=1}^{m}(m-s+1) Z_{d_{s}} \\
\forall d_{s} \in Q-\bigcup_{i=1}^{s-1} k_{i-1} \\
Q=\left\{\begin{array}{c}
1,2, \ldots \ldots \mathrm{m} \\
k_{0}=\emptyset \\
\mathrm{s}=1,2, \ldots \ldots \mathrm{m}
\end{array}\right.
\end{gathered}
$$

Therefore, through the construction of the two-stage model, it is finally possible to obtain the global optimal solution, with the smallest sum of the order picking cost and the waiting cost, under the intensive mobile shelf of a single moving channel.

\section{Two-stage model algorithm design for order picking}

From the previous analysis, we can see the minimum picking time cost problem under our assumption is a NP hard problem ${ }^{[6]}$. That means it is generally difficult to find an accurate optimal solution. However, the genetic algorithm combined with Matlab software could helpful to solve this problem ${ }^{[7]}$. The specific algorithm is designed as follow:

\subsection{Coding}

(1) we first randomly sequence a mobile channel that needs to be opened. Then take this sequencing as a gene. For example, sequencing the channels that need to be opened according to order $B_{d}$, could be transformed as, sequencing all the non 0 variables in the set $\left\{E_{d 1}, E_{d 2}, \cdots, E_{d n}\right\}$, which is a gene;

(2) On the basis of the previous step, we could get $m$ genes corresponding to $m$ orders .

\subsection{Generating an initial population}

As shown in Table 3, the initial population is generated by decimal coding, and there is a total of $p$ individuals, each of which has $m$ genes, and each gene has a number of elements between 0 and $n$.

Table 3 the initial population

\begin{tabular}{c|c}
\hline serial number & Picking order \\
\hline 1 & $\{(1,3 \ldots),(2,5, \ldots), \ldots\}$ \\
\hline 2 & $\{(2,8 \ldots),(6,9, \ldots), \ldots\}$ \\
\hline$\ldots \ldots$ & \multicolumn{2}{|c}{$\ldots \ldots$} \\
\hline$p$ & $\{(7,2 \ldots),(1,5, \ldots), \ldots\}$ \\
\hline
\end{tabular}

\subsection{Determine the fitness function}

The fitness function at this stage is:

$$
W=\sum_{s=1}^{m}(m-s+1) Z_{d_{s}}
$$

Calculate the size of the individual's fitness, that is, the time spent on each order picking in the order in which the different channels are opened.

\subsection{Individual evaluation: fitness check}

The fitness of $p$ individuals was calculated in turn, as shown in Table 4.

Table 4 fitness of individuals

\begin{tabular}{c|c|c|c|c|c}
\hline Individual serial number & 1 & 2 & 3 & $\ldots \ldots$ & $\mathrm{p}$ \\
\hline Adaptability & $W_{1}$ & $W_{2}$ & $W_{3}$ & $\ldots \ldots$ & $W_{p}$ \\
\hline
\end{tabular}

\subsection{Copy, transfer, variation}

(1) Arranging the various project according to the serial number, we can find the value of the fitness of each individual ${ }^{[8]}$. According to the order of the individual serial numbers, the cumulative value $K_{c}$ of the obtained fitness can be calculated as $K_{c}=W_{1}+W_{2}+\cdots+W_{p}$. Meanwhile, as shown in Table 5, random number $R_{c}$ between 0 and $K_{p}$ could be generated and the total number of $R_{c}$ is $\mathrm{p}$ ( $\mathrm{p}$ is a positive integer large enough) ${ }^{[9]}$.

Table 5 variable of copy, cross and variation

\begin{tabular}{c|c|c|c|c|c|c|c}
\hline $\begin{array}{c}\text { serial number } \\
(1 \leq \mathrm{c} \leq \mathrm{p})\end{array}$ & 1 & 2 & 3 & 4 & 5 & $\ldots$ & $\mathrm{p}$ \\
\hline Adaptability: $W$ & $W_{1}$ & $W_{2}$ & $W_{3}$ & $W_{4}$ & $W_{5}$ & $\ldots$ & $W_{p}$ \\
\hline $\begin{array}{c}\text { Accumulated } \\
\text { value of fitness: } K_{\varepsilon}\end{array}$ & $K_{1}$ & $K_{2}$ & $K_{3}$ & $K_{4}$ & $K_{5}$ & $\ldots$ & $K_{p}$ \\
\hline $\begin{array}{c}\text { Random number } \\
\text { between } 0 \text { and } \\
K_{p}: R_{c}\end{array}$ & $R_{1}$ & $R_{2}$ & $R_{3}$ & $R_{4}$ & $R_{5}$ & $\ldots$ & $R_{p}$ \\
\hline
\end{tabular}

The individual copy rules are as follows:

(1) Compare the values of $R_{1}$ with the values from $K_{1}$ to $K_{p}$, one by one. The first $K_{c}$ that is greater than $R_{1}$ is selected, and the corresponding individual serial number is copied once;

(2) Compare the values of $R_{2}$ with the values from $K_{1}$ to $K_{p}$, one by one. The first $K_{c}$ encountered greater than $R_{2}$ is selected, and the corresponding individual serial number is copied once;

(3) and so on, until the number of individuals copied reaches $\mathrm{p}$, then the copy is stopped.

(2) Do non-put back random sampling until we got the schemes numbers more the 0.3 times $m$, then do the following operations:

(1) randomly select two elements inside each gene in each sorting scheme, and exchange the pair of elements positions ;

(2) Randomly select a picking scheme, randomly select two gene in this scheme, and exchange the positions of the two genes.

(3) Until all the selected schemes have been done.

\subsection{Termination conditions}


The termination condition is 50 times. We need to find the position where the fitness function value is optimal and stable and take this solution as the optimal solution of the problem ${ }^{[10]}$.

\section{6. simulation samples}

There is a set of 30 rows movable shelves, which has one moving channel that can be opened. Its initial state is opened at $Y_{5}$. There is a batch of 50 orders, and the specific channel conditions that each order needs to open are represented by a randomly generated $50 \times 30 \quad 0-1$ matrix. The sum of the position distance of the moving channel and the waiting cost of each order will be selected as the observed variables ${ }^{[11]}$. Based on Matlab software, the genetic algorithm program is written for this problem, and the target is to find optimal value with a lowest time $\operatorname{cost}^{[12]}$. The specific procedure is like the following:

Intensive mobile shelf two-stage order picking model genetic algorithm program

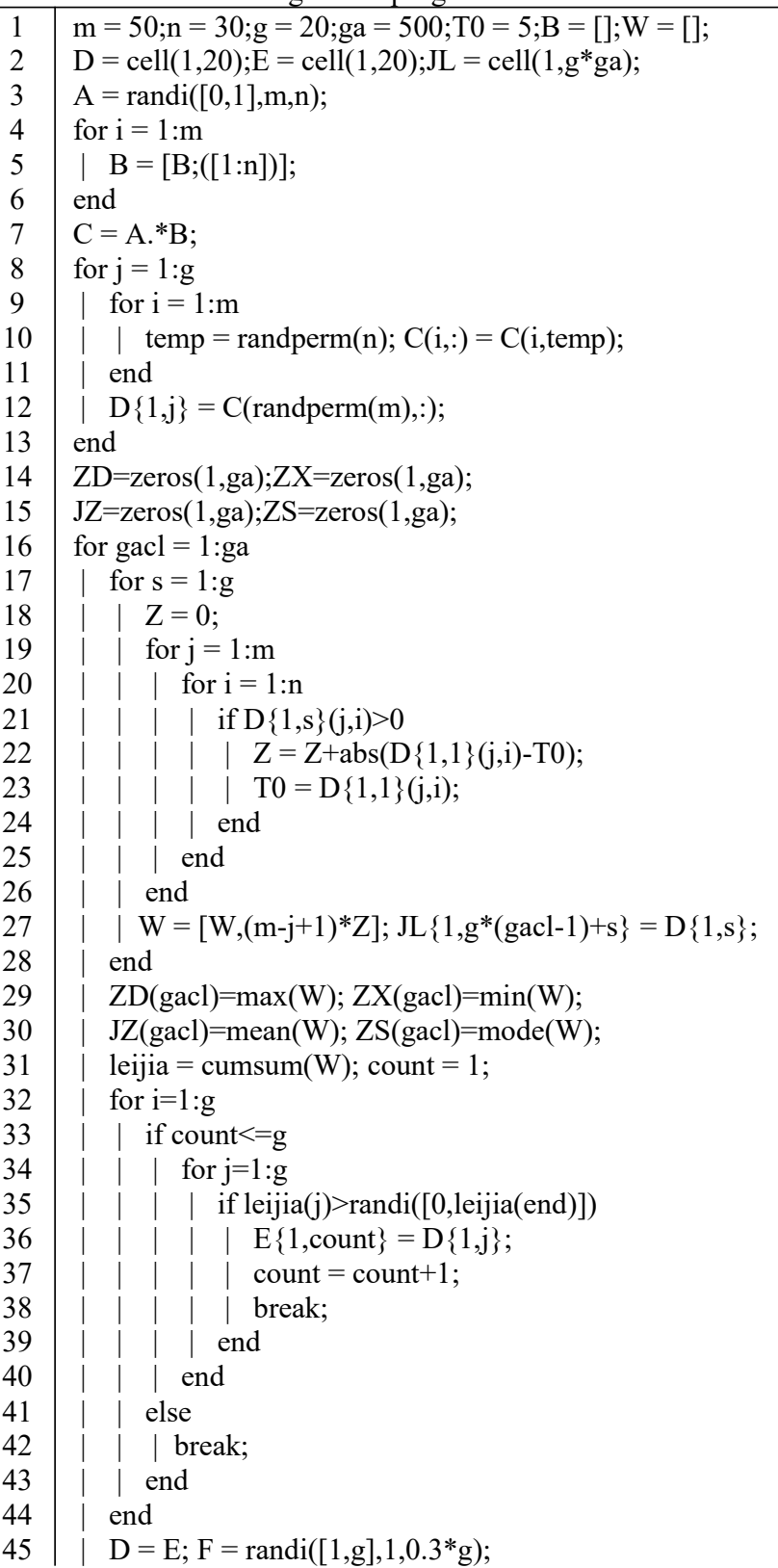

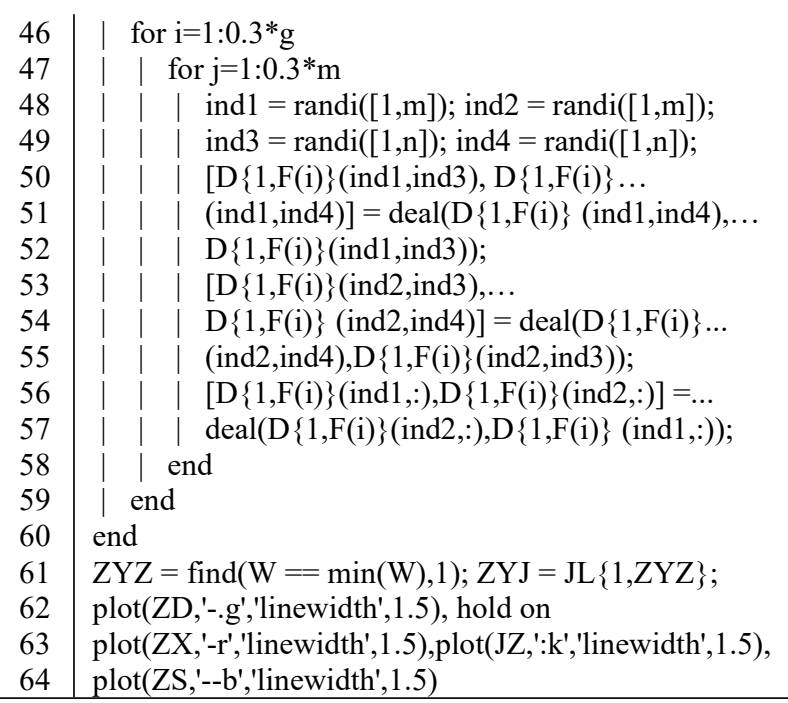

By running the above program, the calculated results are shown in Figure 4. The optimal solution of the previous example is found at the 99th iteration, and the optimal value of the corresponding fitness function is 7270 , which is the sum of the moving shelf position distance and the waiting cost involved in the example problem. The total time cost required for the batch of orders can be derived from the time required for each position of the shelf to move.

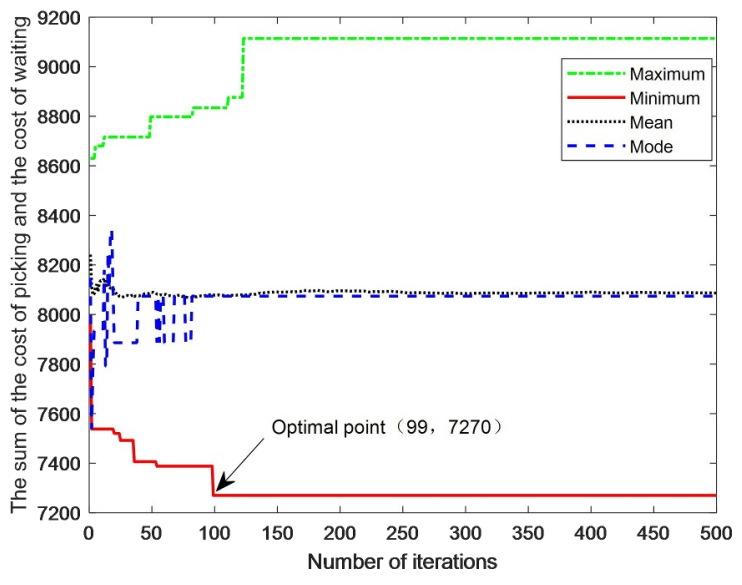

Figure 4 calculation results

Starting from the fitness function to achieve the optimal calculation results, the optimal picking order of 50 orders in this example can be further traced, as shown in Table 6, which is an optimal solution for the research problem.

Table 6 results of picking order

\begin{tabular}{c|c|c|c|c|c}
\hline Picking & Order & Picking & Order & Picking & Order \\
\hline 1 & O29 & 18 & O9 & 35 & O2 \\
\hline 2 & O31 & 19 & O28 & 36 & O16 \\
\hline 3 & O24 & 20 & O50 & 37 & O23 \\
\hline 4 & O14 & 21 & O44 & 38 & O7 \\
\hline 5 & O38 & 22 & O46 & 39 & O13 \\
\hline 6 & O33 & 23 & O35 & 40 & O42 \\
\hline 7 & O15 & 24 & O32 & 41 & O21 \\
\hline 8 & O1 & 25 & O20 & 42 & O11 \\
\hline 9 & O12 & 26 & O25 & 43 & O45 \\
\hline 10 & O19 & 27 & O22 & 44 & O10 \\
\hline
\end{tabular}




\begin{tabular}{c|c|c|c|c|c}
\hline 11 & O8 & 28 & O43 & 45 & O17 \\
\hline 12 & O18 & 29 & O5 & 46 & O27 \\
\hline 13 & O4 & 30 & O48 & 47 & O6 \\
\hline 14 & O49 & 31 & O40 & 48 & O41 \\
\hline 15 & O39 & 32 & O26 & 49 & O30 \\
\hline 16 & O36 & 33 & O37 & 50 & O34 \\
\hline 17 & O3 & 34 & O47 & & \\
\hline
\end{tabular}

In addition, through further analysis of the output results of Matlab, if opening the shelves with a random sequencing, the time cost could be very high. Even if only concern the lowest channel opening time without optiming the order selection sequencing, the mean and maximum value of the fitness will reach 8100 and 9150 respectively. The model constructed in this paper can not only greatly reduce the time cost of picking activities in a completely random state, but also use the genetic algorithm to further optimize the order picking sequencing based on the optimization of each order picked. The time cost of the whole batch of orders is further reduced by $10 \sim 25 \%$. In addition, as the scale of intensive mobile shelves increases and the number of orders increases, the optimization effect will be more significant.

\section{Conclusions and prospects}

In summary, with the application of intensive mobile shelves, while improving the utilization of storage space, it also puts forward higher requirements for order picking activities. Based on the analysis of the application and research status of intensive mobile shelves, this paper studies the selection of bulk orders under single-channel intensive mobile shelves from the following aspects:

(1) Under the single-channel intensive mobile shelf, the sorting problem of single order is transfer into TSP ;

(2) Construct a two-stage model and take the minimum total cost of order picking and waiting as the objective in a single period;

(3) Based on the basic idea of genetic algorithm, the algorithm is designed in detail and implemented by Matlab programming.

(4) The validity of the model and the solution method are further proved by an example.

Future research can be expanded from the following aspects:

(1) Concern the mathematical model, there may be more than one algorithm and more than one optimal solution for this kind of problem. How to improve the algorithm and find these optimal solutions more quickly and effectively can become a further study topic;

(2) This problem is a NP hard problem. The complexity of the problem will increase exponentially with the increase of the scale of the intensive mobile shelves and the number of orders. For the problems with a very large scale, we need to find more suitable algorithm;

(3) the assumptions impact a lot on the model. It is necessary to make more adjustments and improvements of the model to fit for different conditions.

\section{References}

1. Wang Cong,Jin Wei,Ma Lin,Wang Wei.Sorting optimization of outsourcing orders for auto parts considering scheduling constraints[J].Industrial Engineering and Management,2016,21(04):106-113.

2. Wu Yingying, Meng Xiangxu, Wang Yanyan, Hu Jinchang. Order Sorting Optimization of "Goods to People" Picking System[J]. Journal of Mechanical Engineering, 2016, 52(04): 206-212.

3. Yu Yangyang. Optimization method of picking path based on genetic algorithm[J]. China Science and Technology Information, 2019(08): 91-94.

4. Shao Zezhen, Dong Baoli. Multi-variety and smallvolume order batching method based on improved genetic K-means algorithm[J].Journal of Zhejiang Sci-Tech University(Natural Science), 2018,39(06):732-738.

5. LI Yongwei,LIU Shul'an,GUO Jinqin.Study on the Optimization of Warehouse Picking Path Based on Double-layer Genetic Algorithm[J].Journal of Taiyuan University(Natural Science),2018,36(02):46-51.

6. Pan Chenghao, Guo Min. Simulation of Batch Picking Path Planning for Warehouse Logistics Robots[J]. Computer and Modernization, 2017(02): 12-16.

7. $\mathrm{Yu}$ Yangyang. Optimization Method of Picking Path Based on Genetic Algorithm[J]. China Science and Technology Information, 2019(08): 91-94.

8. Shao Zezhen, Dong Baoli. Multi-variety and small batch order batching method based on improved genetic K-means algorithm[J].Journal of Zhejiang Sci-Tech University(Natural Science), 2018,39(06):732-738.

9. Li Yongwei, Liu Shuan, Guo Jinqin. Research on Warehouse Picking Path Optimization Based on Double-layer Genetic Algorithm[J]. Journal of Taiyuan University: Natural Science Edition, 2018, 36(02): 46-51.

10. Pan Chenghao, Guo Min. Simulation of Batch Picking Path Planning for Warehouse Logistics Robots[J]. Computer and Modernization, 2017(02): 12-16.

11. He Yi. Optimization of order picking path in logistics distribution center under genetic algorithm[J].Commercial and Economic Research,2016(21):110-111.

12. Xia Li, Hou Shiwang, Liu Jun. Research on multilane picking operation scheduling of single stacker based on hybrid algorithm[J]. Industrial Engineering, 2016, 19(05): 33-38. 